\title{
Development of multiplex real-time PCR assays for differential detection of capripoxvirus, parapoxvirus, and foot-and-mouth disease virus
}

\author{
Amaresh Das ${ }^{1}$, Yin Wang ${ }^{2}$, Shawn Babiuk ${ }^{3}$, Jianfa Bai ${ }^{4}$, Kimberly Dodd $^{1}$, and Wei Jia ${ }^{1}$ \\ ${ }^{1}$ US Department of Homeland Security Plum Island Animal Disease Center \\ ${ }^{2}$ Kansas State University College of Veterinary Medicine \\ ${ }^{3}$ Affiliation not available \\ ${ }^{4}$ Kansas State University
}

September 16, 2020

\begin{abstract}
This study reports the development of multiplex real-time PCR assays for differential detection of capripoxvirus (CaPV), parapoxvirus (PaPV), and foot-and-mouth disease virus (FMDV) in sheep, goats and cattle. Three multiplex assays were developed, a capripox (CaP) rule-out assay for simultaneous detection and differentiation of CaPV and PaPV, a FMD rule-out assay for simultaneous detection and differentiation of FMDV and PaPV, and a FMD/CaP rule-out assay for simultaneous detection and differentiation of CaPV, PaPV and FMDV. All multiplex assays included ?-actin gene ACTB as an internal positive control to monitor PCR inhibition and accuracy of nucleic acid extractions. The optimized assays were highly specific to the target viruses ( $\mathrm{CaPV}, \mathrm{PaPV}$, and FMDV) with no cross-reactivity against other differential viruses. Using positive control plasmids as template, the limit of detection (LOD) of the multiplex assays were estimated as 2 (CaPV), 7 (PaPV), and 15 (FMDV) copies per assay. The amplification efficiency (AE) and correlation co-efficient (R2), estimated from the standard curves (Ct vs. $\log 10$ template dilution), were $94-106 \%$ and $>0.99$, respectively, for CaP and FMD rule-out assays, $96-116 \%$ (AE) and $>0.98$ (R2), respectively, for CaP/FMD rule-out assays and 91-102\% and >0.99, respectively, for the corresponding singleplex assays. The diagnostic sensitivity (DSe) of the multiplex assays was assessed on 35 (CaPV), 36 (PaPV) and 39 (FMDV) clinical specimens collected from experimentally (CaPV and FMDV) and naturally (PaPV) infected animals, and all tested positive (DSe 100\%) except two FMDV specimens that were tested negative (37/39; DSe 95\%). The newly developed multiplex assays offer a valuable tool for differential detection of clinically indistinguishable CaPV, PaPV, and FMDV in suspected animals and animals with mixed infections.
\end{abstract}

\begin{abstract}
This study reports the development of multiplex real-time PCR assays for differential detection of capripoxvirus (CaPV), parapoxvirus (PaPV), and foot-and-mouth disease virus (FMDV) in sheep, goats and cattle. Three multiplex assays were developed, a capripox $(\mathrm{CaP})$ rule-out assay for simultaneous detection and differentiation of $\mathrm{CaPV}$ and $\mathrm{PaPV}$, a FMD rule-out assay for simultaneous detection and differentiation of FMDV and PaPV, and a FMD/CaP rule-out assay for simultaneous detection and differentiation of CaPV, PaPV and FMDV. All multiplex assays included $\beta$-actin gene $A C T B$ as an internal positive control to monitor PCR inhibition and accuracy of nucleic acid extractions. The optimized assays were highly specific to the target viruses (CaPV, PaPV, and FMDV) with no cross-reactivity against other differential viruses. Using positive control plasmids as template, the limit of detection (LOD) of the multiplex assays were estimated as 2 (CaPV), 7 (PaPV), and 15 (FMDV) copies per assay. The amplification efficiency (AE) and correlation coefficient $\left(R^{2}\right)$, estimated from the standard curves (Ct vs. $\log _{10}$ template dilution), were $94-106 \%$ and $>0.99$,
\end{abstract}


respectively, for CaP and FMD rule-out assays, $96-116 \%(\mathrm{AE})$ and $>0.98\left(\mathrm{R}^{2}\right)$, respectively, for CaP/FMD rule-out assays and $91-102 \%$ and $>0.99$, respectively, for the corresponding singleplex assays. The diagnostic sensitivity (DSe) of the multiplex assays was assessed on 35 (CaPV), 36 (PaPV) and 39 (FMDV) clinical specimens collected from experimentally ( $\mathrm{CaPV}$ and FMDV) and naturally (PaPV) infected animals, and all tested positive (DSe 100\%) except two FMDV specimens that were tested negative (37/39; DSe 95\%). The newly developed multiplex assays offer a valuable tool for differential detection of clinically indistinguishable CaPV, PaPV, and FMDV in suspected animals and animals with mixed infections.

\section{INTRODUCTION}

Capripoxvirus (CaPV), parapoxvirus (PaPV), and foot-and-mouth disease virus (FMDV) are etiological agents of capripox $(\mathrm{CaP})$, parapox $(\mathrm{PaP})$, and foot-and-mouth disease $(\mathrm{FMD})$, respectively. These viruses are common pathogens infecting ruminants including sheep, goats and cattle. $\mathrm{PaP}$ is endemic in many countries worldwide including the United States, while CaP and FMD are endemic in many regions of Asia and most of Africa. Both CaP and FMD are listed as economically important foreign animal disease (FAD) in the US and reportable to the World Organization for Animal Health, formerly the Office International des Epizooties (OIE).

CaPVs belong to the genus Capripoxvirus within the familyPoxviridae, subfamily Chordopoxvirinae and comprised of three genetically similar species:Sheeppoxvirus(SPV), Goatpoxvirus (GPV), and Lumpy skin disease virus(LSDV) (Tuppurainen et al. 2017). Animals susceptible to SPV, GPV, and LSDV are sheep, goats, and cattle, respectively. There has never been a CaP outbreak in the Western hemisphere; however, the disease is endemic in many parts of the world, including many regions of Asia (SP, GP and LSD), Africa (SP, GP, and LSD) with recent outbreaks in Southeast Europe (LSD) that were successfully controlled through vaccination. In endemic countries, the diseases cause large economic losses in production and trade. The clinical presentations of CaPV infections include fever, depression, reduced milk production (cow), and skin lesions (nodule, papules, and macules) (Babiuk et al. 2008). Disease is mainly transmitted by insect vectors (LSDV), direct contact of infected animals and/or through aerosols (SPV and GPV) (Tuppurainen et al. 2017). LSDV infections in cattle have a moderate morbidity rate (5-45\%) and a low mortality rate (below 10\%) while SPV and GPV infections (sheep and goats) have high morbidity rate (70-90\%) and a high mortality rate (up to $50 \%$ ).

PaPVs belong to the family Poxviridae and genusParapoxvirus and consists of four species: Orf virus(ORFV), Bovine papular stomatitis virus (BPSV),Pseudocowpox virus (PCPV) and Parapoxvirus of red deer in New Zealand (PVNZ) (Buttner and Rziha 2002). Ruminants are the main hosts of PaPVs worldwide, including sheep and goats (infected by ORFV) and cattle (infected by BPSV or PCPV). All PaPVs are closely related genetically and antigenically, exhibit similar morphology, and have virulence mechanisms that are indistinguishable (Fleming et al. 1993). PaPV infections are often mild; and the clinical disease is characterized by fever, sores and proliferative dermatitis including raised/elevated skin, and ulcerative lesions on leg, mouth, teats, lips, nostrils, gums and tongue. In most cases, the infected animals recover completely by themselves within 1-2 months without any post-infection treatment (Haig and Mercer 1998; McKeever et al. 1988). PaPVs are also zoonotic, infecting humans with lesions characterized by large, painful nodules (milker's nodule) on the hands after close contact with skin lesions of infected animals or handling virus-contaminated materials (Memer \& Tyring 1995; Handler et al. 2017). Although PaP is not a reportable disease to OIE, its clinical resemblance to $\mathrm{CaP}$ and FMD requires a differential diagnosis of the disease to rule-out $\mathrm{CaP}$ and FMD.

FMDVs belong to the family Picornaviridae and genus Aphthovirus (Grubman and Baxt 2004) and consists of seven distinct serotypes: A, Asia1, C, O, and SAT (Southern African Territories) -1, -2, and -3 (Alexandersen et al., 2003; Bachrach 1968). It is a highly contagious viral disease affecting wild and domestic cloven-hoofed animals, including pigs and ruminants (Alexandersen et al., 2003; Arzt et al., 2011b) and is a reportable disease to OIE. FMD has a substantial impact on the economy of endemic countries due to international trade restrictions in animal products. The disease spreads rapidly by direct and indirect contact with infected animals or an infected environment (Alexandersen et al., 2003). The disease is endemic in many regions 
of the world including Africa, most of Asia and South America with sporadic outbreaks in free areas. An outbreak of FMD in countries that are free from the disease can result in massive economic losses due to culling of large numbers of animals and restrictions in the movement of livestock and trade in animal products. The clinical presentation of FMD is characterized by fever, reduced milk production, lameness and vesicular lesions on tongue, muzzle, feet and teats (Artz et al. 2011a \& 2011b). Infected sheep and goats can shed the virus with mild or no clinical disease which is often overlooked allowing FMDV to be undetected (Barnett \& Cox 1999; Callens et al. 1998; Hughes et al. 2002).

Mixed infections with multiple viral pathogens are common and it can be devastating if any of the co-infecting pathogens is highly contagious, such as FMDV or CaPV. Mixed infections of FMDV or CaPV with other viruses in ruminants have been reported, including PaPV (Xu et al. 2019; He et al. 2017; Venkatesan et al. 2014a \& 2014b; Chu et al. 2011), peste des petits ruminant virus (PPRV) (Kumar et al. 2016), and blue tongue virus (BTV) (Malik et al. 2011). Clinical diagnosis of mixed infections involving CaPV, PaPV, and FMDV can be challenging due to very similar clinical signs associated with these diseases. In countries free of $\mathrm{CaP}$ and FMD but endemic to PaP, it is extremely important to detect and differentiate PaPV from FMDV and CaPV in suspected animals. Differential diagnosis of these viruses is more challenging in countries where all three viruses are endemic.

In this study, three multiplex real-time PCR (qPCR) and RT-PCR (RT-qPCR) assays were developed for the differential detection of CaPV, PaPV and FMDV. The performance of the newly developed assays were compared against the corresponding singleplex assays to evaluate their sensitivity, specificity, and compatibility.

\section{MATERIALS AND METHODS}

\subsection{Viruses and specimens}

All viruses used in this study were either cell culture (CC) or clinical specimen (CS) (swabs, tissues and blood). Multiple CC isolates of the three CaPV members (LSDV, GPV and SPV) and the seven serotypes of FMDV (A, Asia1, C, O, SAT1, SAT2 and SAT3) were used. Other CC viruses include bovine herpes virus (BHV) types 1 and 2, PPRV, bovine viral diarrhea virus (BVDV), vesicular stomatitis virus (VSV) strains New Jersey (VSV-NJ) and Indiana (VSV-IN), and BTV serotypes 1, 6, 9, 13 and 22. All CC viruses were obtained from the biological repository of the Reagents and Vaccine Services Section (RVSS) of the USDA Foreign Animal Disease Diagnostic Laboratory (USDA-FADDL), located at the Plum Island Animal Disease Center (PIADC), Orient Point, New York, USA. The complete list of the viruses and their origins are shown in supplemental table 1. Clinical specimens used in the study were as follows: thirty five CaPV specimens (swabs, skin lesions, skin nodules, scab, EDTA blood) from sheep $(n=10)$, goats $(n=15)$ and cattle $(\mathrm{n}=10)$, experimentally infected with SPV-Nigeria, GPV-India and LSDV-Neethling, respectively, obtained from the Canadian Food Inspection Agency (CFIA), Winnipeg, Canada, as previously reported (Das et al. 2017b); thirty-six PaPV specimens (skin and scabs) from naturally infected sheep ( $\mathrm{n}=9$ ), goats $(\mathrm{n}=15)$, and cattle $(\mathrm{n}=12)$, obtained from the Diagnostic Services Section (DSS) of FADDL (DSS-FADDL) as previously reported (Das et al. 2017a); and thirty-nine FMDV specimens (tissues, EDTA blood and serum) from experimentally infected cattle, obtained from USDA Agricultural Research Services (USDAARS), PIADC. The PaPV specimens were collected from infected ruminants (sheep, goats and cattle) from different geographical regions of the United States between 2003 and 2005 that were submitted to DSSFADDL to rule-out CaPV and FMDV. Negative control specimens (skin) were also used, and they were collected from healthy cattle.

\subsection{Viral RNA/DNA extractions}

Viral RNA (FMDV, VSV, BTV and PPRV) was extracted from $140 \mu$ l of CC or CS using the RNeasy Mini Kit (Qiagen, Valencia, CA) according to the manufacturer's instructions. Viral DNA (CaPV, PaPV and BHV) was extracted from $100 \mu \mathrm{l}$ of CC or CS using the DNeasy Blood and Tissue Kit (Qiagen) according to the manufacturer's instructions. Tissue and skin samples were processed to $10 \%$ homogenates in Dulbecco's Modified Eagle medium (DMEM, Thermo Fisher Scientific) as described previously (Das et al. 2017a). To 
minimize template degradation, extracted viral DNA/RNA were appropriately diluted in nuclease-free water and aliquoted into a smaller volume for one time use only and stored at $-70{ }^{\circ} \mathrm{C}$.

\subsection{Singleplex qPCR and RT-qPCR assays}

All singleplex (one target) assays reported in this study were carried out on ABI 7500 Fast thermocycler (Thermo Fisher Scientific). The OIE recommended real-time PCR assays were used for the detection of CaPV (Balinsky et al. 2008) and FMDV (Callahan et al. 2002), and a recently published qPCR assay (Das et al. 2017a) was used for the detection of PaPV. The beta actin gene $A C T B$, ubiquitously expressed in all mammalian species, including ruminants (sheep, goat and cow) was used as an internal positive control (IPC), and amplified as described previously (Das et al. 2017b). The original CaPV and FMDV assays were slightly modified by replacing their PCR chemistries as reported earlier (Das et al. 2017b; Wang et al. 2020). The qPCR assays were carried out using Path-ID qPCR Mastermix (Thermo Fisher Scientific), and the RTqPCR assays were carried out using Path-ID Multiplex One-Step RT-PCR Kit (Thermo Fisher Scientific). The TaqMan probes used in qPCR/RT-qPCR were labeled with FAM (CaPV or PaPV) or VIC (ACTB ) as the reporter dye and TAMRA (FMDV and PaPV) or MGBNFQ (CaPV) as the quencher dye. A complete list of the primers and probes used in the PCR assays are shown in supplemental table 2. The composition of the PCR mastermixes included 1x kit-suppled buffer (mastermix), forward primer (FP), reverse primer $(\mathrm{RP})$, probe $(\mathrm{Pr})$ and $2 \mu \mathrm{l}$ of template (viral DNA or RNA), plus balance nuclease-free water to a final volume of $25 \mu \mathrm{l}$. The concentrations of the primers and probe used in the PCR mastermixes were: $0.25 \mu \mathrm{M}$ $\mathrm{FP}, 0.625 \mu \mathrm{M}$ RP and $0.25 \mu \mathrm{M}$ Pr for CaPV qPCR; $0.2 \mu \mathrm{M}$ FP, $0.2 \mu \mathrm{M}$ RP and $0.2 \mu \mathrm{M}$ Pr for PaPV or $A C T B$ qPCR and $0.2 \mu \mathrm{M}$ FP, $0.2 \mu \mathrm{M}$ RP and $0.2 \mu \mathrm{M}$ Pr for FMDV RT-qPCR. The thermocycling conditions for qPCR (CaPV, PaPV or $A C T B$ ) included 1 cycle of $95{ }^{\circ} \mathrm{C}$ for 10 min followed by 45 cycles of $95{ }^{\circ} \mathrm{C}$ for 15 sec and $65{ }^{\circ} \mathrm{C}$ for $1 \mathrm{~min}$. The thermocycling conditions for RT-qPCR (FMDV) included one cycle of $45{ }^{\circ} \mathrm{C}$ 10 min (RT step) and then 1 cycle of $95{ }^{\circ} \mathrm{C}$ for 10 min followed by 45 cycles of $95{ }^{\circ} \mathrm{C}$ for 15 sec and $65{ }^{\circ} \mathrm{C}$ for $1 \mathrm{~min}$.

\subsection{Multiplex qPCR and RT-qPCR assays}

Three multiplex assay designs were tested and optimized: 1) a capripox (CaP) rule-out (3-plex) assay for simultaneous detection and differentiation of CaPV and PaPV, 2) a FMD rule-out (3-plex) assay for simultaneous detection and differentiation of PaPV and FMDV, and 3) a CaP/FMD rule-out (4-plex) assay for simultaneous detection and differentiation of CaPV, PaPV and FMDV. All multiplex assays included $A C T B$ ( $\beta$ actin) as the IPC. The final selection of the reporter/quencher dyes for the TaqMan probes were: FAM/MGBNFQ (CaPV), Cy5/IADQ (PaPV) and VIC/QSI ( $A C T B$ ) for CaP rule-out assays; FAM/MGBNFQ (FMDV), Cy5/IADQ (PaPV) and VIC/QSI (ACTB ) for FMD rule-out assays; and FAM/MGBNFQ (CaPV), Texas Red/IADQ (FMDV), Cy5/IADQ (PaPV) and VIC/QSI ( $A C T B$ ) for FMD/CaP rule-out assays. The composition of the PCR mastermixes and the thermocycling conditions used in the multiplex assays were the same as used in the corresponding singleplex assays (above), except for FMDV where the primers/probe concentrations were doubled.

\subsection{Artificial templates used as positive amplification control (PAC)}

The artificial templates used as PAC were cloned recombinant plasmids carrying PCR amplicon of the target viruses. The PACs used for the CaPV (Das et al. 2012) and PaPV (Das et al. 2017a) qPCR assays were reported earlier. The PAC used for the FMDV RT-qPCR assay was an in-vitrotranscribed RNA derived from a cloned plasmid as described previously (Wang et al. 2020). The purified PACs (plasmid DNA or in-vitrotranscribed RNA) were quantified on NanoDrop 2000c spectrophotometer (Thermo Fisher Scientific) and used as template in the LOD (limit of detection) studies.

\subsection{Analytical sensitivity: singleplex assays}

The LOD of the singleplex assays was determined using serial dilutions of the PACs (plasmid DNA or invitro transcribed RNA) or the viral DNA/RNA in nuclease-free water as template. The viral DNA/RNA extracted from CC grown viruses were used as template except for PaPV which was extracted from CS of 
an infected cattle (FADDL/DSS accession ID 15-020595). The LOD of the PACs were expressed as copy number per assay where each copy represents one genome copy of the virus particle. The copy number was calculated using the Avogadro's number (6.022 $\times 10^{23}$ molecules/mole) using the following formula:

Copy number $=\left[\right.$ mass $\left.(\mathrm{g}) \times 6.022 \times 10^{23}\right] /\left[\right.$ length $\left.(\mathrm{bp}) \times 10^{9} \times 650 \mathrm{Da}\right]$, or, using the web link http://cels.uri.edu/gsc/cndna.html. When using the viral DNA/RNA as template, the LODs were expressed either as the highest detectable dilution or the lowest detectable virus titer $\left(\mathrm{TCID}_{50} / \mathrm{ml}\right)$ per assay.

The amplification efficiency (AE) and correlation coefficient $\left(\mathrm{R}^{2}\right)$ of the multiplex assays were determined from the standard curves prepared by plotting the fluorescence threshold cycle $(\mathrm{Ct})$ values against $\log _{10}$ serial dilution of the PACs or the viral DNA/RNA used as template.

\subsection{Analytical sensitivity: multiplex assays}

The LOD of the multiplex assays was determined using $A C T B$ as an IPC. To include $A C T B$ in the multiplex assays, the template (PAC or the viral DNA/RNA) was serially diluted in the DNA extracted from the skin of healthy cattle, also referred to as negative skin DNA. The LODs were expressed either as copy number (PACs) or the highest detectable dilution (viral DNA/RNA) or the lowest detectable virus titer $\left(\mathrm{TCID}_{50} / \mathrm{ml}\right)$ per assay as described above (2.6).

\subsection{Analytical specificity and diagnostic sensitivity: multiplex assays}

The analytical specificity was assessed against both target and differential viruses. The CaP rule-out assays were tested against the target (CaPV) viruses using CC isolates of LSDV, GPV and SPV and CS of PaPV (BPSV and ORFV), and differential viruses using CC isolates of BHV and PPRV. The FMD rule-out assays were tested against the target (FMDV) viruses using CC isolates of the seven serotypes of FMDV (A, Asia1, C, O, SAT1, SAT2 and SAT3), CS of PaPV (BPSV and ORFV), and differential viruses using CC isolates of BVDV, VSV and BTV.

The DSe of the multiplex assays was assessed against thirty-five CaPV, thirty-six PaPV and thirty-nine FMDV specimens from experimentally infected (CaPV and FMDV) or naturally infected (PaPV) animals as described in the materials and methods.

\section{RESULTS}

\subsection{Optimization of the multiplex assays}

The original CaPV, PaPV and FMDV assays (above) used in the study were optimized and validated on low throughput PCR platform Cepheid SmartCycler (Sunnyvale, CA), which can run a maximum of up to sixteen reactions for each machine run. In this study, the above assays were further optimized on the high throughput PCR platform ABI 7500 Fast which can run a maximum of 96 reactions per run. For performance evaluation, parallel singleplex assays were performed on SmartCycler and ABI 7500 Fast using serial dilutions of viral DNA/RNA as template. The estimated LOD (highest detectable dilution) of each target (CaPV, PaPV or FMDV) remained the same between the two PCR platforms (results not shown).

Several reporter and quencher dyes (fluorophores) were tested for optimization of the multiplex assays and the best results were obtained using FAM, Cy5, Texas Red and VIC as reporter dyes and MGBNFQ (minor groove binding non fluorescent quencher, Thermo Fisher Scientific), IBDQ (Iowa Black ${ }^{\circledR}$ dark quencher, IDT) and QSY (Thermo Fisher Scientific) as quencher dyes. The quencher dye TAMRA, used in the original singleplex assays (PaPV, FMDV, and $A C T B$ ), was found unsuitable for multiplex assays as it interfered with the fluorescence spectra of other reporter dyes resulting in inconsistent $\mathrm{Ct}$ values. Therefore, TAMRA was replaced with other quencher dyes (above). Optimized performance of the multiplex assays were obtained using FAM (CaPV or FMDV), Cy5 (PaPV) and VIC (ACTB ) as reporter dyes for CaP and FMD rule-out assays, and FAM (CaPV), Cy5 (PaPV), Texas Red (FMDV) and VIC ( $A C T B$ ) for CaP/FMD rule-out assays (see results below).

\subsection{Analytical sensitivity using PACs as template}


The PACs (cloned plasmid or in-vitro transcribed RNA) were serially diluted in nuclease-free water (singleplex) or in negative skin DNA (multiplex) and used as template. In the CaP rule-out assays, the CaPV PAC was co-amplified with $A C T B$ in the presence of primers and probe of PaPV; and in the FMD rule-out assays, the FMDV PAC (transcribed RNA) was co-amplified with $A C T B$ in the presence of primers and probe of $\mathrm{PaPV}$. The PaPV PAC was subjected to two multiplex rule-out assays, PaP1 and PaP2. In PaP1, the PAC was co-amplified with $A C T B$ in the presence of primers and probe of $\mathrm{CaPV}$; and in PaP2, the PAC was co-amplified with $A C T B$ in the presence of primers and probe of FMDV. The LODs (copy number/assay), estimated from the standard curves (Ct vs. $\log _{10}$ template dilutions), were 2 (CaPV), 7 (PaPV) and 15 (FMDV) by both singleplex and multiplex assays (Figs 1A, 1B and 1C). The $\mathrm{AE}$ and $\mathrm{R}^{2}$ values, calculated from the standard curves were: $96-110 \%$ (AE) and $>0.99\left(\mathrm{R}^{2}\right)$, respectively, for singleplex assays; and 94-106\% (AE) and >0.99 ( $\left.\mathrm{R}^{2}\right)$, respectively, for multiplex assays, regardless of origin of the PACs. These results show linearity of amplification of the PACs by the multiplex assays with minimal interference from the primers and probes of the non-target assays including the $A C T B$.

\subsection{Analytical sensitivity using viral DNA/RNA as template}

Combined CaPV and PaPV DNAs, mixed at equal ratio, were serially diluted in negative skin DNA and used as template for the CaP rule-out assays; and combined PaPV DNA and FMDV RNA, mixed at equal ratio, were serially diluted in negative skin DNA and used as template for the FMD rule-out assays. The estimated LODs (highest detectable dilution per assay) calculated from the standard curves (Ct vs. $\log _{10}$ template dilutions) were: $10^{6}$ (LSDV, GPV, SPV and PaPV), by CaP rule-out and the corresponding singleplex assays (Table 1 \& Fig 2); $10^{6}$ (PaPV, FMDV Asia1, C, SAT1 and SAT3), by FMD rule-out and the corresponding singleplex assays (Table $2 \&$ Figure 3), and $10^{7}$ (FMDV A, O, and SAT2), by FMD rule-out and the corresponding singleplex assays (Table $2 \&$ Figure 3$)$. The LODs, with respect to virus titers $\left(\mathrm{TCID}_{50} / \mathrm{ml}\right.$ per assay) were: between $10^{0.3}$ and $10^{1.6}$ for LSDV, GPV and SPV, by CaP rule-out assays (Table 1), and between $10^{0.45}$ and $10^{1.8}$ for FMDV serotypes A, Asia1, C, O, SAT1, SAT2 and SAT3, by FMD rule-out assays (Table 2). The $\mathrm{AE}$ and $\mathrm{R}^{2}$ values, calculated from the standard curves (Ct vs. $\log _{10}$ template dilutions) were $94-106 \%$ (AE) and $>0.99\left(\mathrm{R}^{2}\right)$, respectively, by CaP rule-out assays (Table 1), and $95-105 \%$ (AE) and $>0.99\left(\mathrm{R}^{2}\right)$, respectively, by FMD rule-out assays (Table 2 ). The AE and $\mathrm{R}^{2}$ values of the multiplex assays are in close agreement with that of the corresponding singleplex assays (Tables $1 \& 2$ ). It should be noted that the LOD (highest detectable dilution per assay) of PaPV by singleplex assays was not shown, which remained same $\left(10^{6}\right)$ as the corresponding multiplex (CaP and FMD rule-out) assays.

For CaP/FMD rule-out assays, combined LSDV and PaPV DNAs were mixed at equal ratio with FMDV-A, FMDV-C or FMDV-O RNA, and serially diluted in negative skin DNA and used as template. The LOD, AE and $\mathrm{R}^{2}$ values were estimated from the standard curves (Supplemental Fig. 1) and the results are summarized in Table 3. The estimated LOD (highest detectable dilution per assay) was $10^{6}$ for all viruses including the FMDV serotypes A and O, which had the LODs dropped by 10-fold compared to the corresponding singleplex and $\mathrm{CaP}$ or FMD rule-out assays $\left(\mathrm{LOD} 10^{7}\right.$ ). The CaP/FMD rule-out assays were also carried out with combined GPV and PaPV or SPV and PaPV DNAs, mixed at equal ratio with FMDV-O or FMDV-C RNA and serially diluted in the negative DNA and used as template. The LOD was $10^{6}$ dilution per assay for all viruses including FMDV-O which was dropped by 10-fold compared to the corresponding singleplex and $\mathrm{CaP}$ or FMD rule-out assays (Supplemental Fig. 2). The $\mathrm{AE}$ and $\mathrm{R}^{2}$ values of the $\mathrm{CaP} / \mathrm{FMD}$ rule out assays, calculated from the standard curves, were 96-116\% (AE) and $>0.98\left(\mathrm{R}^{2}\right)$, respectively, which are very close to their acceptable ranges (AE 90-110\%; $R^{2}>0.99$ ) (Table 3; Supplemental Figs $1 \& 2$ ).

\subsection{Specificity, cross-reactivity and diagnostic sensitivity of the multiplex assays}

The specificity of the multiplex assays was evaluated against multiple isolates/species of the target as well as differential viruses. The CaP rule-out assays were evaluated against multiple CC isolates of LSDV, GPV and SPV, 36 CS (PaPVs), and multiple CC isolates of the non-target viruses BHV and PPRV. The FMD rule-out assays were evaluated against multiple CC isolates of the seven serotypes of FMDV (A, Asia1, C, O, SAT1, SAT2 and SAT3), 36 CS (PaPVs), and multiple CC isolates of the non-target viruses BVDV, VSV and BTV. The results show $100 \%$ specificity of the CaP rule-out (Table 4) and FMD rule-out (Table 5) 
assays with no cross-reactivity against any of the non-target differential viruses.

The DSe of the multiplex assays was assessed on CS from experimentally (CaPV and FMDV) and naturally (PaPV) infected animals. Thirty-five CaPV and $36 \mathrm{PaPV}$ (BPSV and ORFV) specimens were tested by CaP rule-out assays and all tested positive (DSe 100\%) (Table 4). Thirty-six PaPV (BPSV and ORFV) and 39 FMDV specimens were tested by FMD rule-out assays, out of which, 37 FMDV specimens (DSe 95\%) and all 36 PaPV specimens (DSe 100\%) tested positive by FMD rule-out assay (Table 5).

\section{DISCUSSION}

A multiplex real-time PCR assay can detect and differentiate two or more pathogens in a single assay, which provides the capacity to diagnose and rule out multiple diseases in a sample containing multiple pathogens. However, the challenge of simultaneous amplification of multiple targets (template) is that asymmetric amplifications can occur, resulting weaker target(s) (lower AE) amplified less efficiently than stronger targets (higher AE). Therefore, it is important to evaluate the performance of the multiplex assays against the corresponding singleplex assays to ensure that there is no loss of sensitivity for each testing target.

In this study, multiplex TaqMan ${ }^{\circledR}$ assays were developed and optimized for the detection and differentiation of CaPV, PaPV and FMDV. The original (singleplex) assays corresponding to each virus were optimized and validated on Cepheid SmartCycler. The above assays were further optimized on high throughput PCR platform ABI 7500 Fast using serial dilutions of viral DNA/RNA as template in this study; and the results were compared against parallel assays ran on Cepheid SmartCycler. The sensitivity of detection (LOD) of the template (viral DNA/RNA) remained unchanged between the two PCR platforms (not shown), indicating comparable performances.

To ensure diagnostic applicability on CS, the newly developed multiplex assays included the host $\beta$-actin gene $A C T B$ as the IPC to detect PCR inhibition and monitor accuracy of nucleic acid extractions. The $\beta$-actin is ubiquitously expressed and detectable in most commonly used CS (skin, tissue, blood and swabs) of all mammalian species including cattle, sheep and goats (Das et al. 2017a \& 2017b), which justifies its inclusion as the IPC. To determine the LOD, the viral DNA/RNA were serially diluted in nuclease-free water (singleplex) or the DNA purified from skin of non-infected healthy animal (multiplex) and used as template. This allowed parallel comparison of the performance of the assays between the singleplex and the multiplex formats. The results (LOD studies) showed no apparent change in the sensitivity of detection of the template (viral DNA/RNA) between the two formats of the assay (singleplex and multiplex), indicating no interference of either the host DNA or the amplification of $A C T B$. The choice of using the DNA purified from skin as a diluent was due to the fact that skin/scab and skin lesions are the most common specimens used for the detection of all three viruses (CaPV, PaPV and FMDV).

Three multiplex assays were developed and optimized: CaP rule-out assays for simultaneous detection and differentiation of $\mathrm{CaPV}$ and PaPV; FMD rule-out assays for simultaneous detection and differentiation of FMDV and PaPV; and CaP/FMD rule-out assays for simultaneous detection and differentiation of CaPV, $\mathrm{PaPV}$ and FMDV. At the onset of the development, the multiplex assays were subjected to the following optimization criteria: concentration of the primers and probe of each target, selection of the reporter and quencher dyes of the TaqMan ${ }^{\circledR}$ probes used for each target, and finally, assessment of the optimized (multiplex) assays against the corresponding singleplex assays. The concentration of the primers and probe of each target used in the multiplex assays remained the same as the corresponding singleplex assays except for FMDV where the primers and probe concentrations were doubled. The selection of the reporter dyes of the TaqMan ${ }^{\mathrm{TM}}$ probes was thoroughly evaluated to rule-out any cross-talk (interference) between the dyes that might adversely affect the sensitivity of the assay. The final selection of the reporter dyes used in the $\mathrm{CaP}$ and FMD rule-out 3-plex assays were FAM (CaPV or FMDV), Cy5 (PaPV) and VIC (ACTB ) and those used in the CaP/FMD rule-out 4-plex assays were FAM (CaPV), Cy5 (PaPV), Texas Red (FMDV) and VIC $(A C T B)$. The use of Texas Red along with FAM and Cy5 as reporter dyes in multiplex TaqMan assays was also reported by others (Fratamico et al. 2011). Preferential use of FAM, Cy5 and VIC in 3-plex assays was also reported by others (Diallo et al. 2011; Del Amo 2013). These dyes (FAM, Cy5 and VIC) have 
wide differences in the excitation and emission wavelengths that reduce the risk of cross-talk or overlapping signals and allow accurate and reliable detection of the template (Cirino et al. 2007; Gunson et al. 2008). The CaP and FMD rule-out (3-plex) assays reported in this study also used FAM, Cy5 and VIC as reporter dyes and exhibited comparable performances (LOD) as the corresponding singleplex assays. The CaP/FMD rule-out (4-plex) assays also exhibited similar performances (LOD) as the corresponding singleplex and CaP and FMD rule out (3-plex) assays for all viruses with the exception of the FMDV serotypes A and O, which showed a 10-fold drop in the LOD. Reduced sensitivity (LOD) of multiplex assays is not unusual as previously reported (Das et al., 2019; Elnifro et al. 2000; Gunson et al. 2008). Reasons for this could be due to preferential amplification of one target (higher AE) over another (lower AE), competition for the same reagents in the mastermix ( $\mathrm{dNPTs}, \mathrm{Mg}^{+2}$ ions etc.) and non-specific interactions between multiple primers sets (Hindson et al. 2008).

The efficacy of the multiplex assays were evaluated against the corresponding singleplex assays using both PACs and the viral DNA/RNA as template. The CaP and FMD rule-out assays exhibited $100 \%$ specificity against all serotypes/isolates of the target viruses (CaPV, PaPV and FMDV) tested, and had no crossreactivity against other viruses including CaPV differentials BHV and PPRV, as well as FMDV differentials BVDV, VSV and BTV. Assessment of DSe of the multiplex assays revealed 100\% DSe against CaPV (35/35) and $\mathrm{PaPV}(36 / 36)$ by CaP rule-out assays; and 100\% DSe against PaPV (36/36) and 95\% DSe against FMDV $(37 / 39)$ by FMD rule-out assays. The two FMDV specimens tested negative by FMD rule-out assay were also tested negative by singleplex FMDV RT-qPCR assay (not shown), indicating comparable DSe between the singleplex and multiplex assays.

Several PCR-based multiplex assays have been reported by others for differential diagnosis of mixed infections involving CaPV, PaPV and FMDV, including conventional PCR/RT-PCR assays (He at al. 2017, Venkatesan et al. 2014a) and multiplex RT-PCR microsphere array assays (Hindson et al. 2008). A duplex (2-plex) TaqMan assay (Venkatesan et al. 2014b) was reported for simultaneous detection and differentiation of SPV, GPV and ORFV but not LSDV or FMDV and the assay was evaluated without using any CS of cattle or IPC. A 4-plex TaqMan assay (Xu et al. 2019) was reported for simultaneous detection and differentiation of SPV, ORFV and FMDV but not GPV or LSDV, and this assay also evaluated without using any CS of cattle or IPC. The newly developed multiplex assays described in this study were evaluated against all three differential viruses and their serotypes (CaPV, PaPV and FMDV) some of which were not included in the above studies. Furthermore, all assays included an IPC $(A C T B)$ to detect PCR inhibition and monitor accuracy of nucleic acid extractions to rule out any false negative results.

The United States has been free from CaP and FMD which is partly due to the implementation of a highly effective FAD surveillance program. The newly developed CaP and FMD rule-out assays can strengthen this program by providing the tools to monitor any incursion of CaPV or FMDV. FADDL has been designated as the only lab in the US allowed to perform FAD diagnosis. Specimens from animals showing clinical signs resembling FADs are routinely sent to FADDL for testing, including those from suspected ruminants infected with PaPV showing clinical signs resembling CaP or FMD. Currently, target specific singleplex assays are carried out to rule-out CaP or FMD, which are time consuming and costly. These singleplex assays potentially can be replaced with the newly developed CaP and FMD rule-out assays. The new assays may be more useful for diagnostics in countries with endemic $\mathrm{CaP}, \mathrm{PaP}$ and FMD, where mixed infections are prevalent. Overall, the newly developed multiplex assays have the potential for use in both routine laboratory diagnosis as well as disease surveillance investigations.

\section{ACKNOWLEDGEMEMNTS}

Authors gratefully acknowledge PIADC USDA Agricultural Research Services (USDA-ARS) for providing FMDV specimens (tissues, EDTA blood and serum) from experimentally infected cattle used in this study.

The findings and conclusions in this preliminary publication are those of the authors and should not be construed to represent any USDA or US Government determination or policy.

\section{ETHICAL APPROVAL}


Animal experiments involving sheep goats and cattle experimentally infected with capripoxvirus were conducted under the approval of the Canadian Science Centre for Human and Animal Health Animal Care Committee, which follows the guidelines of the Canadian Council on Animal Care.

\section{CONFLICT OF INTEREST}

The authors declare that they have no conflict of interests.

\section{data availability statement}

The data that support the findings of this study are available from the corresponding author upon reasonable request.

\section{REFERENCES}

Alexandersen, S., Zhang, Z., A. Donaldson, A. I., \& Garland, A. J. M. (2003). The Pathogenesis and Diagnosis of Foot-and-Mouth Disease.Journal of Comparative Pathology . 129, 1-56. https://doi.org/10.1016/S0021-9975(03)00041-0

Arzt, J., Baxt, B., Grubman, M. J., Jackson, T., Juleff, N., Rhyan, J., Reider, E., Waters, R., \& Rodriguez, L. L. (2011a). The pathogenesis of foot-and-mouth disease II: viral pathways in swine, small ruminants, and wildlife; myotropism, chronic syndromes, and molecular virus-host interactions. Transboundary and Emerging Diseases . 58, 305-326.https://doi.org/10.1111/j.1865-1682.2011.01236.x

Arzt, J., Juleff, N., Zhang, Z, \& Rodriguez, L. L. (2011b). The pathogenesis of foot-and-mouth disease I: viral pathways in cattle. Transboundary and Emerging Diseases , 58, 291-304.https://doi.org/10.1111/j.18651682.2011.01204.x

Babiuk, S., Bowden, T. R., Boyle, D. B., Wallace, D. B., \& Kitching, R. P. (2008). Capripoxviruses: An Emerging Worldwide Threat to Sheep, Goats and Cattle. Transboundary and Emerging Diseases . 55, 263-272.https://doi.org/10.1111/j.1865-1682.2008.01043.x

Bachrach, H. L. 1968. Foot-and-mouth disease. Annual Review of Microbiology . 22,

201-244. 10.1146/annurev.mi.22.100168.001221

Balinsky, C. A., Delhon, G., Smoliga, G., Prarat, M., French, R. A., Geary, J., Rock, D. L., \& Rodriguez, L. L. (2008). Rapid preclinical detection of sheeppox virus by a real-time PCR assay. Journal of Clinical Microbiology , 46, 438-442. https://doi.org/10.1128/JCM.01953-07

Barnett, P. V., \& Cox, S. J. (1999). The role of small ruminants in the epidemiology and transmission of foot-and-mouth disease. Veterinary Journal , 158, 6-13. https://doi.org/10.1053/tvjl.1998.0338

Buttner, M, \& Rziha, H. J. (2002). Parapoxviruses: from the lesion to the viral genome. Journal of veterinary medicine. B, Infectious diseases and veterinary public health , 49, 7-16.

https://doi.org/10.1046/j.1439-0450.2002.00539.x

Callahan J. D., Brown, F., Osorio, F.A., Sur J. H., Kramer, E., Long, G. W., Lubroth, J., Ellis, S. J., Shoulars, K. S., Gaffney, K. L., Rock, D. L., \& Nelson, W. M. (2002). Use of a Portable Real-Time Reverse Transcriptase Polymerase Chain Reaction Assay for Rapid Detection of Foot-and-Mouth Disease Virus. Journal of American Veterinary Medical Association, 220, 1636-1642.

https://doi.org/10.2460/javma.2002.220.1636

Callens, M., De Clercq, K., Gruia, M. \& Danes, M. (1998). Detection of foot-and-mouth disease by reverse transcription polymerase chain reaction and virus isolation in contact sheep without clinical signs of foot-andmouth disease. Veterinary Quarterly, 20 (Suppl. 2), 37-40. https://doi.org/10.1080/01652176.1998.9694964 
Chu, Y., Yan, X., Gao, P., Zhao, P., He, Y., Liu, J. \& Lu, Z. (2011). Molecular detection of a mixed infection of Goatpox virus, Orf virus, and Mycoplasma capricolum subsp. capripneumoniae in goats. Journal of Veterinary Diagnostic Investigation, 23, 786-789.

https://doi.org/10.1177/1040638711407883

Cirino, N. M., Tavakoli, N. P., Madison-Anetnucci, S., Egan, C. 2007. Multiplex RT-PCR in microbiology. In: Mackay IM (ed), Real time PCR in microbiology: from diagnosis to characterization. Caister Academic Press, p. 183-221. ISBN: 978-1-913652-35-7

Das, A., Xu, L., \& Jia, W. (2019). Development of conventional and real time PCR assays for rapid species authentication of mammalian cell lines commonly used in veterinary diagnostic laboratories. Research in Veterinary Science, 126, 170-177. https://doi.org/10.1016/j.rvsc.2019.08.029

Das, A., Babiuk, S., \& McIntosh, M. T. (2012). Development of a Loop-Mediated Isothermal Amplification Assay for Rapid Detection of Capripoxviruses. Journal of Clinical Microbiology , 50, 1613-1620. https://DOI.org/10.1128/JCM.06796-11

Das, A., Ward, G., Lowe, A., Xu, L., Moran, K., Renshaw, R., Dubovi, E., Resising, M., \& Jia, W. (2017a). Development and validation of a highly sensitive real-time PCR assay for rapid detection of Parapoxviruses. Journal of Veterinary Diagnostic Investigation , 29, 499-507.

https://doi.org/10.1177/1040638716680676

Das, A., Deng, M. Y., Babiuk, S., \& McIntosh, M. T. (2017b). Modification of two capripoxvirus real time PCR assay to improve diagnostic sensitivity and include beta-actin as an internal positive control. Journal of Veterinary Diagnostic Investigation, 29, 351-356.

https://doi.org/10.1177/1040638717695609

Del Amo, J., Sotelo, E., Fernandez-Pinero, J., Gallardo, C., Llorente, F., Aguero, M., Jimenez-Clavero, M. A. (2013) A novel quantitative multiplex real-time RT-PCR for the simultaneous detection and differentiation of West Nile virus lineages 1 and 2, and of Usutu virus.Journal of Virological Methods , 189, 321-327. https://doi.org/10.1016/j.jviromet.2013.02.019

Diallo, I. S., Corney, B. G., \& Rodwell, B. J. (2011). Detection and differentiation of bovine herpesvirus 1 and 5 using a multiplex real-time polymerase chain reaction, Journal of Virological Methods, 175, 46-52. https://doi.org/10.1016/j.jviromet.2011.04.013

Elnifro, E. M., Ashshi, A. M., Cooper, R. J., \& Klapper, P.E. (2000). Multiplex PCR: optimization and application in diagnostic virology. Clinical Microbiology Review , 13, 559-570.

https://doi.org/10.1128/CMR.13.4.559

Fleming, S. B., Blok, J., Fraser, K. M., Mercer, A. A., \& Robinson, A. J. (1993). Conservation of genetic structure and arrangement between vaccinia virus and orf virus. Virology, 195, 175-184.

https://doi.org/10.1006/viro.1993.1358

Fratamico, P. M., Bagi, L. K., Cray, W. C. Jr., Narang, N., Yan, X., Medina, M., \& Liu, Y. (2011). Detection by Multiplex Real-Time Polymerase Chain Reaction Assays and Isolation of Shiga ToxinProducingEscherichia coli Serogroups O26, O45, O103, O111, O121, and O145 in Ground Beef. Foodborne Pathogens and Disease , 8, 601-607.

https://doi.org/10.1089=fpd.2010.0773

Grubman, M. J., \& Baxt, B. (2004). Foot-and-mouth disease.Clinical microbiology reviews , 17, 465-493. https://doi.org/10.1128/CMR.17.2.465-493.2004 
Gunson, R. N., Bennett, S., Maclean, A. \& Carman, W. F. (2008). Using multiplex real time PCR in order to streamline a routine diagnostic service. Journal of Clinical Virology , 43, 372-375. https://doi.org/10.1016/j.jcv.2008.08.020

Haig, D. M., \& Mercer, A. A. 1998. Ovine diseases, Orf. Veterinary Research , 29, 311-326.

PMID: 9689744

Handler, N. S., Handler, M. Z., Rubins, A., Rubins, S., Septe, M., Janninger, C. K., \& Schartz, R. A. (2017). Milker's nodule: an occupational infection and threat to the immunocompromised.Journal of European Academy Dermatology and Venereology, 32, 537-541.

https://doi.org/10.1111/jdv.14687

He, Y-P., Zhang, Q., Fu, M-Z. \& Xu, X-G. (2017). Development of multiplex PCR for simultaneous detection and differentiation of six DNA and RNA viruses from clinical samples of sheep and goats. Journal of Virological Methods, 243, 44-49.

https://doi.org/10.1016/j.jviromet.2017.01.012

Hindson, B. J., Reid, S. M., Baker, B. R., Ebert, Katja, Ferris, N. P., Bentley Tammero, L. F., Lenhoff, R. J., Naraghi-Arani, P., Vitalis, E. A., Slezak, T. R., Hullinger, P. J., \& King, D. P. (2008). Diagnostic evaluation of multiplexed reverse transcription-PCR microsphere array assay for detection of Foot-and-mouth and lookalike disease viruses. Journal of Clinical Microbiology , 46, 1081-1089. https://doi.org/0.1128/JCM.01740-07

Hughes, G. J., Mioulet, V., Kitching, R. P., Woolhouse, M. E., Alexandersen, S. \& Donaldson, A. I. (2002). Foot-and-mouth disease virus infection of sheep: implications for diagnosis and control. Veterinary Record, $150,724-727$.

https://doi.org/10.1136/vr.150.23.724

Kumar, N., Barua, S., Riyesh, T., Chaubey1, K. K., Rawat, K. D, Khandelwal, N., Mishra, A. K., Sharma, N., Chandel, S. S., Sharma, S., Singh, M. K., Sharma, D. K., Singh, S. V., Tripathi, B. N. (2016). Complexities in Isolation and Purification of Multiple Viruses from Mixed Viral Infections: Viral Interference, Persistence and Exclusion, PLoS One, 11(5):e0156110. doi: 10.1371.

Malik, Y. S., Singh, D., Chandrashekar, K. M., Shukla, S., Sharma, K., Vaid, N., \& Chakravarti, S. (2011). Occurrence of Dual Infection of Peste-Des-Petits-Ruminants and Goatpox in Indigenous Goats of Central India. Transboundary and Emerging Diseases , 58, 268-273.

https://doi.org/10.1111/j.1865-1682.2011.01201.x

McKeever, D. J., Jenkinson, D. M., Hutchinson, G., \& Reid, H. W., (1988). Studies of the pathogenesis of orf virus infection in sheep. Journal of Comparative Pathology . 99, 317-328.

https://doi.org/10.1016/0021-9975(88)90052-7

Memar, O., \& Tyring, S.K. (1995). Cutaneous viral infections. Journal of American Academy of Dermatology . 33, 279-287.https://doi.org/10.1016/0190-9622(95)90262-7

Tuppurainen, E. S. M., Venter, E. H., Shisler, J. L., Gari, G., Mekonnen, G. A., Juleff, N., Lyons, N. A., De Clercq, K., Upton, C., Bowden, T. R., Babiuk, S., \& Babiuk, L. A. (2017). Review: Capripoxvirus Diseases: Current Status and Control. Transboundary and merging Diseases . 64, 729745.https://doi.org/10.1111/tbed.12444

Venkatesan, G., Balamurugan, V., \& Bhanuprakash, V. (2014a). Multiplex PCR for simultaneous detection and differentiation of sheeppox, goatpox and orf viruses from clinical samples of sheep and goats. Journal of Virological Methods, 195, 1-8.

https://doi.org/10.1016/j.jviromet.2013.10.009 
Venkatesan, G., Balamurugan, V., \& Bhanuprakash, V. (2014b). TaqMan based real-time duplex PCR for simultaneous detection and quantification of capripox and orf virus in clinical samples. Journal of Virological Methods , 201, 44-50. https://doi.org/10.1016/j.jviromet.2014.02.007

Wang, Y., Das, A., Zheng, W., Porter, E., Xu, L., Noll, L., Liu, X., Dodd, K., Jia, W., \& Bai, J. (2020). Development and evaluation of multiplex real-time RT-PCR assays for the detection and differentiation of foot-and-mouth disease virus and Seneca Valley virus 1.Transboundary and Emerging Diseases , 67, 604616.https://doi.org/10.1111/tbed.13373

Xu, X., Yang, F., Zhang, Q., Xu, Y., Huang, J., Fu, M., \& Zhang, W. (2019). Development of a multiplex TaqMan qPCR assay for simultaneous detection and differentiation of four DNA and RNA viruses from clinical samples of sheep and goats. Journal of Virological Methods , 266, 58-64. https://doi.org/10.1016/j.jviromet.2019.01.015

\section{Figure legends}

Figure 1

LOD of CaPV, PaPV and FMDV PACs by singleplex and multiplex assays. A, CaPV PAC serially diluted in nuclease-free water (1-plex) or in negative skin DNA in the presence (3-plex) or absence (2-plex) of PaPV qPCR primers and probe; B, FMDV PAC (reverse transcribed RNA) serially diluted in nuclease-free water (1-plex) or in negative skin DNA in the presence (3-plex) or absence (2-plex) of PaPV qPCR primers and probe; C, PaPV PAC serially diluted in nuclease-free water (1-plex) or in negative skin DNA in the absence (2-plex) or presence of CaPV qPCR (PaP1, 3-plex) or FMDV RT-qPCR (PaP2, 3-plex) primers and probe. Amplification of $A C T B$ shown in the graph was taken from 3-plex assays only.

Figure 2

LOD of LSDV, GPV, SPV and PaPV by singleplex (1-plex) and CaP rule-out (3-plex) assays. The viral DNA was serially diluted in nuclease-free water (1-plex) or negative skin DNA (3-plex) and used as template. A, serial dilution of LSDV (1-plex) or combined LSDV/PaPV (3-plex); B, serial dilutions of GPV (1-plex) or combined GPV/PaPV (3-plex); C, serial dilutions of SPV (1-plex) or combined SPV/PaPV (3-plex). Not shown in the figure is the LOD of PaPV DNA by singleplex assays which remained same $\left(10^{6}\right.$ dilution $)$ as the corresponding CaP rule-out assays.

Sources of the viral DNAs: LSDV (LSDV-Uganda), GPV (GPV-Pendik) and SPV (SPV-HELD) DNAs were extracted from CC grown viruses (Supplemental Table 1). The PaPV (BPSV) DNA was extracted from CS of a naturally infected cattle (FADDL/DSS accession ID 15-020595).

Figure 3

LOD of PaPV and the seven serotypes of FMDV by singleplex (1-plex) and FMD rule-out (3-plex) assays. The viral DNA/RNA was serially diluted in nuclease-free water (1-plex) or negative skin DNA (3plex) and used as template. A, serial dilution of FMDV-A (1-plex) or combined PaPV/FMDV-A (3-plex); $\mathrm{B}$, serial dilution of FMDV-Asia1 (1-plex) or combined PaPV/FMDV-Asia1 (3-plex); C, serial dilution of FMDV-C (1-plex) or combined PaPV/FMDV-C (3-plex); D, serial dilution of FMDV-O (1-plex) or combined PaPV/FMDV-O (3-plex); E, serial dilution of FMDV-SAT1 (1-plex) or combined PaPV/FMDV-SAT1 (3plex); F, serial dilution of FMDV-SAT2 (1-plex) or combined PaPV/FMDV-SAT2 (3-plex); G, serial dilution of FMDV-SAT3 (1-plex) or combined PaPV/FMDV-SAT3 (3-plex). Not shown in the figure is the LOD of PaPV DNA by singleplex RT-qPCR assays which remained same $\left(10^{6}\right.$ dilution $)$ as the corresponding FMD rule-out (3-plex) assays.

Sources of the viral DNA/RNA: FMDV RNAs of the serotypes A (A23 Kenya), Asia1 (PAK 1/54), C (C3 RESENDE), O (O1 Manisa), SAT1 (Rhodesia), SAT2 (Zimbabwe 5/81) and SAT3 (South Africa) were extracted from CC grown viruses (Supplemental Table 1). The source of the PaPV DNA was the same as described in the legends to Figure 2. 
Supplemental Figure 1

LOD of CaPV, PaPV and FMDV by CaP/FMD rule-out assay. A, combined LSDV/PaPV/FMDV-A (DNA/RNA) serially diluted in negative skin DNA; B combined LSDV/PaPV/FMDV-C serially diluted in negative skin DNA; C. combined LSDV/PaPV/FMDV-O serially diluted in negative skin DNA.

Supplemental Figure 2

LOD of CaPV, PaPV and FMDV by CaP/FMD rule-out assay. A, combined GPV/ PaPV/FMDV-C (DNA/RNA) serially diluted in negative skin DNA; B, combined GPV/PaPV/FMDV-O (DNA/RNA) serially diluted in negative skin DNA; C, combined SPV/ PaPV/FMDV-C (DNA/RNA) serially diluted in negative skin DNA; D, combined SPV/PaPV/FMDV-O (DNA/RNA) serially diluted in negative skin DNA.
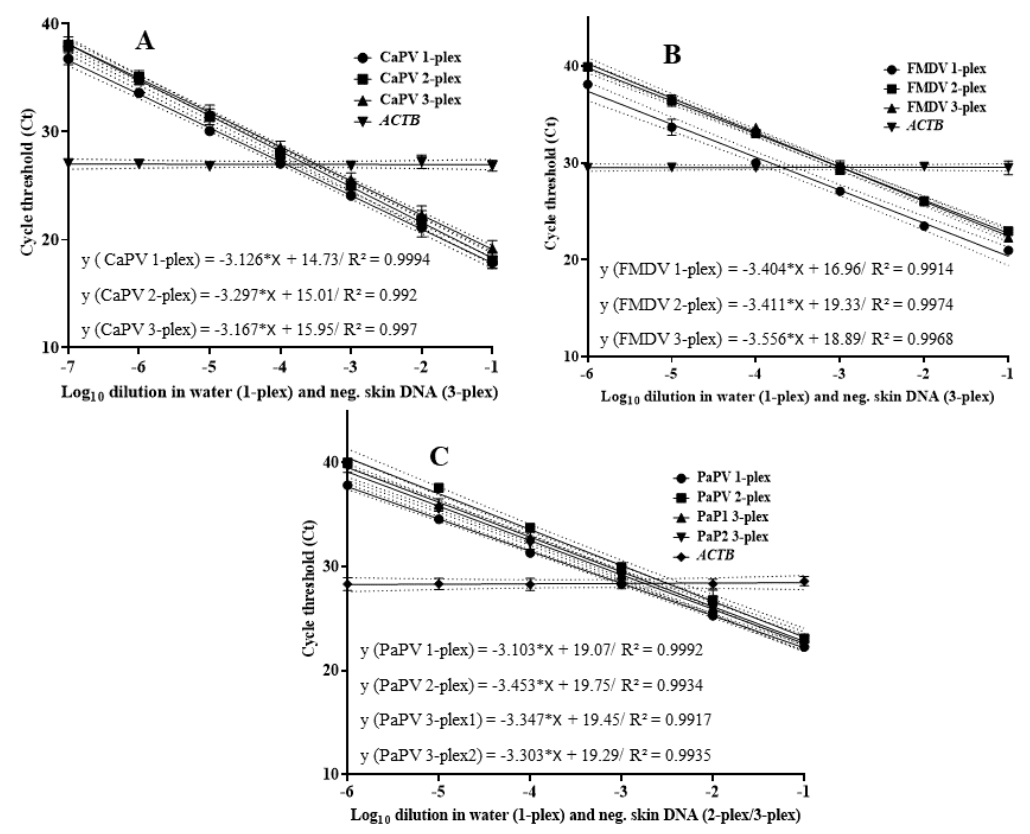

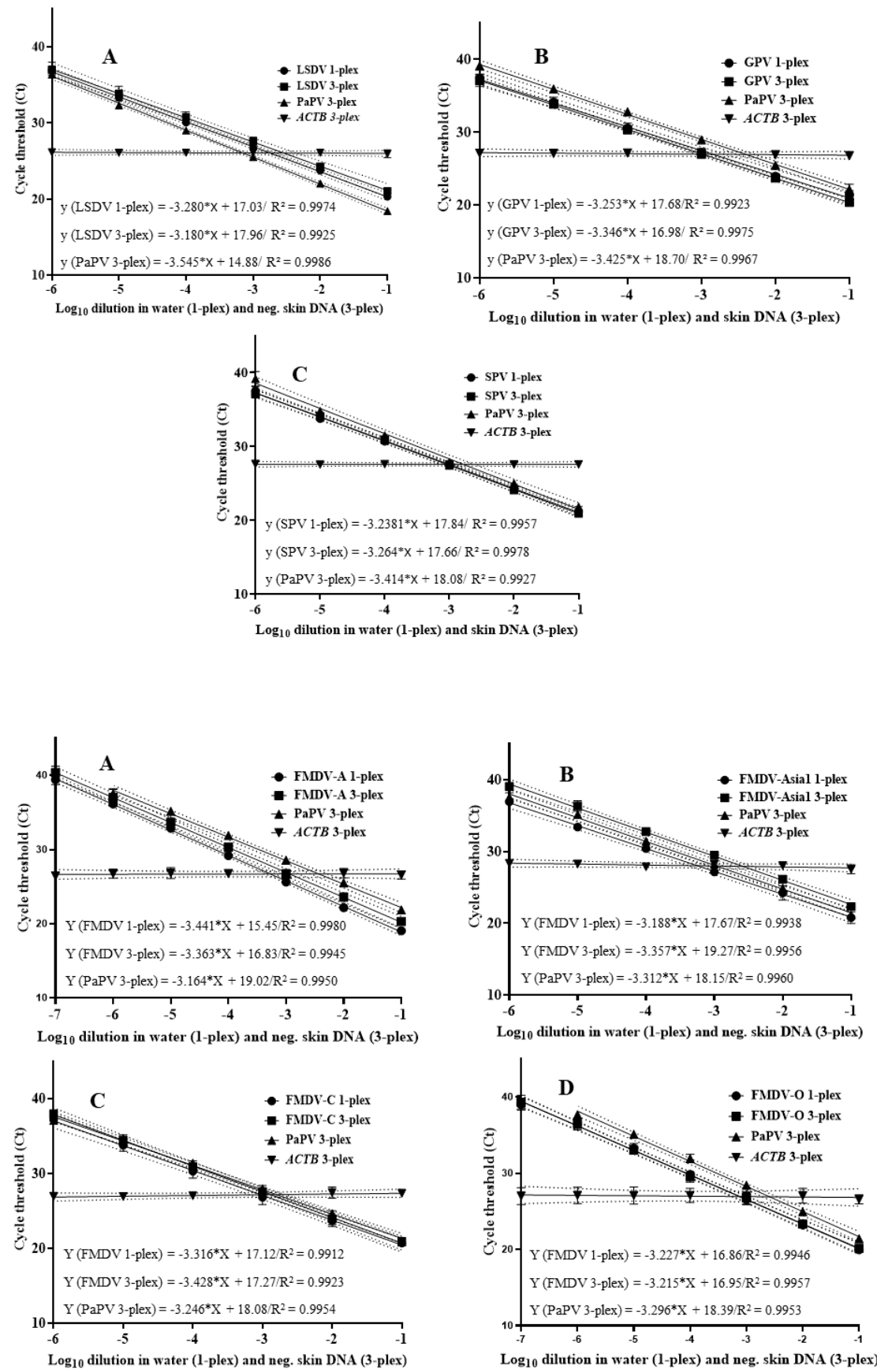

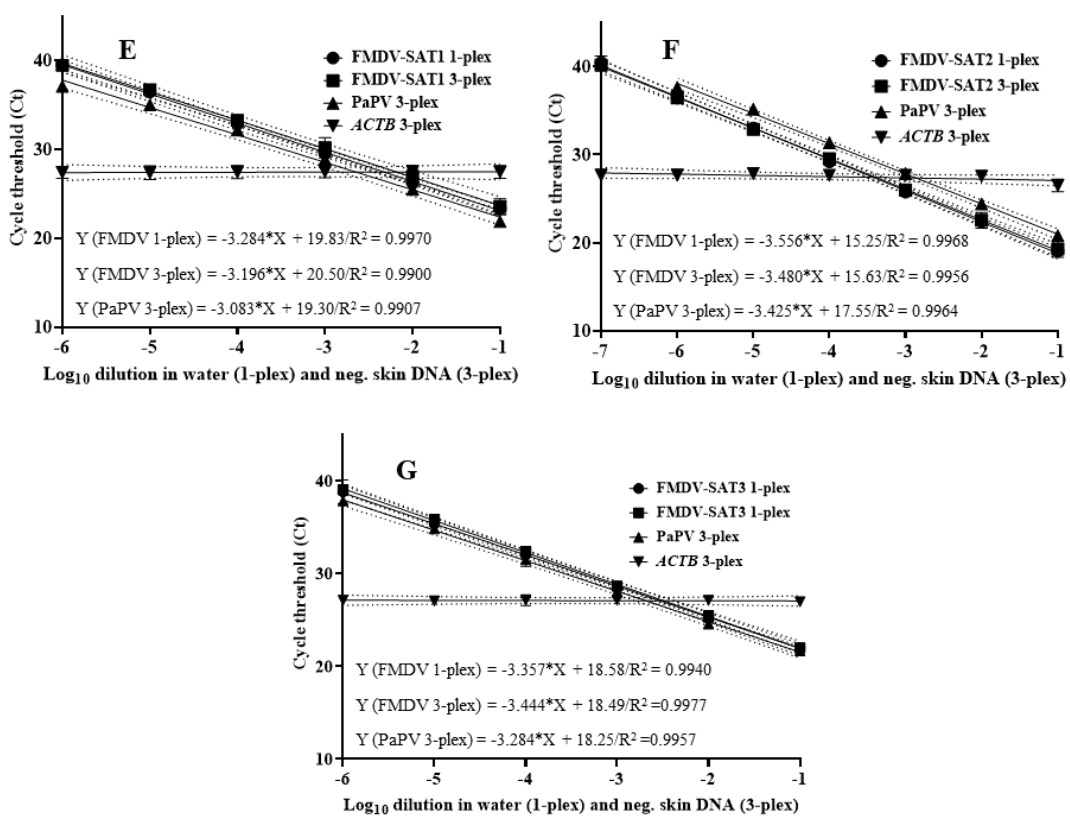

\section{Hosted file}

Table 1.docx available at https://authorea.com/users/359362/articles/481410-development-ofmultiplex-real-time-pcr-assays-for-differential-detection-of-capripoxvirus-parapoxvirusand-foot-and-mouth-disease-virus

\section{Hosted file}

Table 2.docx available at https://authorea.com/users/359362/articles/481410-development-ofmultiplex-real-time-pcr-assays-for-differential-detection-of-capripoxvirus-parapoxvirusand-foot-and-mouth-disease-virus

\section{Hosted file}

Table 3.docx available at https://authorea.com/users/359362/articles/481410-development-ofmultiplex-real-time-pcr-assays-for-differential-detection-of-capripoxvirus-parapoxvirusand-foot-and-mouth-disease-virus

\section{Hosted file}

Table 4.docx available at https://authorea.com/users/359362/articles/481410-development-ofmultiplex-real-time-pcr-assays-for-differential-detection-of-capripoxvirus-parapoxvirusand-foot-and-mouth-disease-virus

\section{Hosted file}

Table 5.docx available at https://authorea.com/users/359362/articles/481410-development-ofmultiplex-real-time-pcr-assays-for-differential-detection-of-capripoxvirus-parapoxvirusand-foot-and-mouth-disease-virus 